\section{Protestkundgebung der Deutschen Gesellschaft für Kinderheilkunde.}

In einer Veröffentlichung des Geschäftsausschusses und ständigen Gutachterausschusses des Deutschen Aerztevereinsbundes vom 11. XII. 1929 ist zum Ausdruck gebracht worden, da $\beta \quad K$ inderärzte Stellen als Schul-, Fürsorge-und Impfärzte nur erhalten sollen, wenn andere Bewerber aus den Reihen der praktischen Aerzte nicht in Betracht k o $\mathrm{mmen}$. Daß Kinderärzte als Fürsorge- und Schulärzte tätig sind, sei nicht erwünscht, da hierbei Schüler und Personen betreut werden, die der den Kinderärzten zustehenden Altersgrenze entwachsen sind. - Diese Bestimmung ist durch eine Mitteilung des gleichen Ausschusses (Deutsches Aerzteblatt 1930 Nr. 8 vom 11. III. 1930) nur temporär außer Kraft gesetzt.

Die Deutsche Gesellschaft für Kinderheilkunde hat diese Auffassung mit größtem Befremden zur Ken his genommen und erhebt energischen Widerspruch dagegen.

Die Bremer Richtlinien, um deren Auslegung es sich in dieser Frage handelt, verbieten lediglich die ärztliche Beratung und Behandlung von Kindern, die das 13. Lebensjahr überschritten haben. Bei der schulärztlichen Tätigkeit ist aber die Behandlung überall ausdrücklich verboten. $E_{S}$ handelt sich hier nur um Beurteilung, Begutachtung und eventuell Zuweisung an andere Aerzte. Und dazu sollten die Kinderärzte nicht befähigt sein? Sie haben die gleiche Ausbildung genossen wie die praktischen Aerzte, dazu kommt noch ihre fachärztliche Ausbildung in der Kinderheilkunde und, nach den Danziger Beschlüssen, sogar noch eine einjährige Ausbildung in innerer Medizin. Sie' sind also in jeder Hinsicht für die Beurteilung aller, auch der älteren Schulkinder besser vorgeschult als die Mehrzahl der praktischen Aerzte. Für die überwiegende Mehrzahl der Personen, die in der Kinder- und Schulfürsorge zu betreuen sind, sind sie aber als ausgesprochene Fachärzte zu bezeichnen, auf die auch die Danziger Beschlüsse zutrefien. Es mutet daher fast grotesk an, sie aus dieser Tätigkeit ausschalten $\mathrm{zu}$ wollen.

Sachliche Gründe lassen sich hierfür nicht vorbringen, und eine solche Verfügung würde daher weder von den Behörden noch von der Oeffentlichkeit verstanden werden.

Die Deutsche Gesellschaft für Kinderheilkunde weist alle derartigen Versuche energisch zurück und verlangt aus sachlichen Gründen vielmehr als Voraussetzung für jede Tätigkeit in der Kinder-und Schulfürsorgeden Nachweiseiner hinreichenden A usbildung in der Kinderheil$\mathrm{k} u \mathrm{nde}$. Wenn sich ein Facharzt für Kinderkrankheiten um eine solche Stelle bewirbt, verdient er im Gegensatz zu den seinerzeit veröffentlichten Richtlinien den Vorzug vor allen anderen Bewerbern.

\section{Der Vorsitzende} gez. I b r a h m.

„Riehtlinien für Stellung und Ausbildung des Sehularztes; aufgestellt von der Deutschen Gesellschaft für Kinderheillkunde auf ihrer 41. ordentlichen Versammlung in Wiesbaden am 13. IV. 1930.

1. Die Schule als Zwangseinrichtung des Staates hat die Aufgabe, sich nicht nur unterrichtlich zu betätigen, sondern auch die Gesundheit der Schüler zu schützen und zu fördern. Die Schule muß bemüht sein, alle Schäden auszuschließen, welche durch den Schulbetrieb entstehen können. Von gleicher Wichtigkeit ist es, die Versammlung der Kinder zu einer gesundheitlichen Sichtung, Beratung und Befürsorgung zu verwenden.

2. Um dieses Ziel zu erreichen, muß das Schularztsystem weit ausgebaut werden. In allen Fragen der Hygiene der Schule und der Gesundheitsförderung der Schüler ist der Arzt maßgebend. Geeignete Räume mit sinnentsprechender Ausstattung für seine Tätigkeit sollen nach Möglichkeit in jeder Schule für seinen Zweck zur Verfügung stehen.

3. Der Schularzt muß innerhalb der einzelnen Schulen zu den Beratungen des Lehrerkollegiums in gesundheitlichen Angelegenheiten hinzugezogen werden. In den Selbstverwaltungskörpern, die sich mit der Schule befassen, muß der Schularzt vertreten sein.

4. Erforderlich ist eine hinreichend große Zahl von Schulärzten mit entsprechender, d. h. dem Zweck angepaßter Ausbildung.
5. Die Grundlage der Ausbildung muß in Anbetracht der Tatsache, daß es sich bei den Schülern ausschließlich um Kinder und Jugendliche handelt, eine ausreichende, etwa 2 Jahre betragende Ausbildung in der Kinderkunde und Kinderheilkunde sein. Fachärzte für Kinderkrankheiten kommen in erster Linie in Betracht.

6. Zur Hauptausbildung soll ein spezieller Kursus der Schulgesundheitspflege von ausreichender Dauer hinzukommen. Während der Dauer dieses Kursus ist auch besonderer Wert auf folgendes zu legen:

1. Seuchenbekämpfung und Sozialhygiene,

2. Psychologie und Psychopathologie des Kindes- und Jugendalters,

3. Physiologie und Pathologie der Leibesübungen.

7. Die Ausbildung der Schulärzte dürfte im Prinzip Angelegenheit der Universitäten und Sozialhygienischen Akademien unter Zuziehung von Praktikern der Schulgesundheitspflege sein.

8. Den jetzt tätigen Schulärzten ist durch besondere Nachschulungskurse Gelegenheit zur Fortbildung zu geben. Außerdem ist darauf zu halten, daß die Schulärzte zu ihrer Fortbildung an geeigneten Veranstaltungen teilnehmen.“" 